\title{
Virtual Reality Technologies in Health Care: A Literature Review of Theoretical Foundations
}

\author{
Michael Knop \\ University of Siegen, Germany \\ michael.knop@,uni-siegen.de \\ Sebastian Weber \\ University of Siegen, Germany \\ sebastian.weber@uni-siegen.de
}

\author{
Caroline Ressing \\ University of Siegen, Germany \\ caroline.ressing@uni-siegen.de \\ Henrik Freude \\ University of Siegen, Germany \\ henrik.freude@uni-siegen.de
}

\author{
Marius Mueller \\ University of Siegen, Germany \\ marius.m.mueller90@gmail.com \\ Bjoern Niehaves \\ University of Siegen, Germany \\ bjoern.niehaves@,uni-siegen.de
}

\begin{abstract}
The digitization of health care promises an improvement of medical care through the adoption of virtual reality (VR) related technologies. Although most undergoing mechanisms of clinical effectiveness are yet not defined theoretically, research approaches have already taken place in several empirical settings. To structure current and upcoming scientific work in this field, we conducted a literature review with regard to theoretical implications of both IS-related and healthcare-related research. We found several theoretical bases to build upon in the field of psychology, but expressed a need for enrichment of theoretical foundations in the field of IS research. We therefore plead for a theoretical foundation enriched by synergetic concepts of clinically effective VR related technologies. Finally, we conclude that VR related technologies appear as a promising approach worth further theoretical and empirical research in order to improve medical care.
\end{abstract}

\section{Introduction}

Digital approaches that enable spatially and temporally independent treatments of patients are receiving increasing scientific, medical, and commercial attention [1]. Especially in times of partially endangered access to care and the unequal distribution of healthcare providers and specialists [2], the comprehensive provision of healthcare services in order to safeguard societal health, patient satisfaction and safety, as well as positive therapy outcomes, requires novel ways of treatment delivery and execution. This increasing need for improved medical care and therapeutic processes represents a major challenge of our time, putting pressure on healthcare providers, who are confronted with high demands, and consumers that struggle with a lacking availability of facilities and professionals. Here, innovative technologies and advancements represent a promising approach to counteract health-related risks [3], maintain or even increase treatment quality and effectiveness [4] and establish new ways of efficient patient treatment [5].

Today, the digitization of healthcare practices and processes exhibits a major momentum. Former manual practices become more and more augmented by digital technologies. Examples appear in the form of virtual, thus spatially independent communication channels between healthcare providers and patients [6], the collection of vital parameters through mobile sensory equipment [7], and novel therapeutic approaches using emerging technologies such as virtual reality (VR) environments [8]. These developments promise a relief of healthcare structures, i.e. medical professionals who are enabled to treat larger amounts of patients, and contribute to a potentially improved treatment quality in the light of declining care availability [9].

One popular technological advancement receiving scientific attention is the development and evaluation of VR related technologies (we subsume virtual, augmented, and mixed reality under this term) and their opportunity to create virtual environments. The application of these technologies represents a promising innovative approach to explore novel ways of digitally supplemented treatments and therapeutic processes decoupled from spatial limitations. Virtual environments have been subject to a plethora of studies in many domains such as (clinical) medicine (e.g., [10]), Information Systems (e.g., [11]), education and learning (e.g., [12]), and robotics (e.g., [13]).

Current VR applications in health care focus on clinical or medical solutions and treatments with regards to pre- or post-operational surgery, psychological 
diseases such as anxiety disorders, stress related disorders, or eating disorders [14-16]. Most of these studies aim at examinations of patient-related outcomes and the effectiveness of treatment catalyzed by VR related technologies [17]. However, research lacks a structured view on the scientific application and theoretical foundation of these technologies. Only few scientific papers include a sound theoretical base in order to explain cause-effect relationships [14, 15]. Already published literature reviews in the field of VR related technologies in health care have already considered development processes and evaluations of VR related technologies [18] from different medical specialties [19] by focusing on specific diseases (e.g., $[20,21])$. Nevertheless, a summary and differentiation of theoretical foundations to explain and predict the effectiveness of VR related technologies is still absent, although a variety of different theoretical approaches are linked to the effectiveness of those technologies, e.g. Cognititive Load Theory for educational purposes. Here, our paper seeks to provide an overview of VR related technologies and their theoretical foundations used in healthcare research. Therefore, our research question (RQ) is the following:

RQ: What are the theoretical foundations linked to clinical effectiveness for the development and use of VR related technologies in healthcare?

To answer our research questions, the remainder of this paper is structured as follows: First, we give an overview of recent VR related technologies and their characteristics. Second, we describe our research method. Thereafter, our main findings with regard to theoretical implications are illustrated. Subsequently, we discuss our finding and derive implications. Finally, some concluding remarks are given.

\section{Background}

\subsection{Characteristics and Advantages of VR related Technologies}

VR technologies offer high potential for educative and intervention-oriented research and practice. Due to specific characteristics, VR head-mounted displays (HMDs) or AR glasses allow its user to experience a totally surrounding and enclosing virtual space or virtually extended environment [22] in which they can interact, create, or manipulate objects [23, 24]. These interactions are possible through the use of controllers or a real-time taken video through a camera [25]. In addition, VR technologies allow a high precision and permission of visualized objects and processes which are too abstract, difficult, intangible, infeasible or impossible to depict in the real world [13]. In turn, this enables users to dive into a virtual environment, i.e. being immersed in an artificial surrounding [26]. The degree of immersion within a virtual space can be distinguished into immersive and non-immersive VR [22]. The latter one refers to desktop-PCs or laptops including two-dimensional (2D) video screen presentations or 2D-rendered images [27]. The first one refers to users wearing complex interface technologies such as VR-HMDs like Oculus Rift or HTC Vive. These technologies are often related to single user experiences [28] and are typically limited to single user sessions of 30 minutes [29]. Briefly summarized, Slater and Sanchez-Vives [13] outline four practical advantages of immersive VR. (I) VR changes abstract settings to tangible ones. For instance, mathematical or geometrical concepts are more easily understandable with immersive VR [12]. (II) VR technology allows its user to be active rather than just passively observing. For example, in surgical training a student learns from practicing instead from observing medical procedures [30]. (III) VR simulations allow users to substitute methods that are desirable by teachers or lecturers but practically infeasible or impossible in reality. For instance, students are able to study different historical or other related important attractions within a short period of time without time or resource restrictions [31, 32]. (IV) VR can break the bounds of reality as a part of exploration. A virtual setting allows to manipulate physical properties such as gravity or making light speed and biological cell utilizing visible [33].

VR related technologies range from fully immersive experiences to unobstructed experiences. Silva et al. [17] describe an extended reality spectrum ranging from virtual reality (VR) to merged reality $(\mathrm{MeR})$ to mixed reality (MR) and augmented reality (AR). These differ from each other in a sense that VR, $\mathrm{MeR}$, and MR consist of interactive virtual objects while AR provides non-interactive virtual objects. Augmented reality brings the computer into the world of the user by augmenting the real environment with virtual objects [34]. Moreover, VR has a virtual background while $\mathrm{MeR}, \mathrm{MR}$, and AR include real backgrounds. For instance, in VR environments the digital replaces the physical, in MR the digital integrates with physical spaces, and in AR the digital is separated from the physical room [17].

\subsection{VR related Applications in Health Care}

Current research and applications with regard to VR related technologies in health care focus on clinical solutions to catalyze or improve routines of medical professionals, such as surgery or body examinations. In general, applications often lack technical standards and theoretical maturity [14, 15]. However, current approaches of VR related technologies in health care are 
promising and address various application fields [17]. For instance, technologies are referred to educational, pre-procedural, therapies/rehabilitation, or intraprocedural purposes [17]. Within educational applications, VR allows to simulate the entire operating environment along with educational material and MR enables multiple users to interact and discuss, while looking at the same educational material such as Virtual Heart [35] or HoloAnatomy [36, 37]. Pre-procedural applications provide its user different images to each eye using specialized glasses to manipulate the onscreen image by a handheld wand $[37,38]$. Within their study, Chan et al. [38] examine cardiologists who evaluated patients who had undergone computed tomography angiography either by AR glasses or a traditional read out. They found that the interpretation time was reduced by usage of AR glasses in comparison to the traditional readout. With regard to inter-procedural visualization, current applications allow users to utilize gesture, gaze, or voice control for sterile control of the display and integrate pre-procedural data in purpose of (real-time) visualizations [39, 40]. A current example for rehabilitation or therapies refers to the idea of combining VR with brain imaging and gaming technologies to retrain the brain to improve upper limb mobility [41]. Kress and Shin [41] found that $90 \%$ of post-stroke patients who used such a system reported an improvement in movement capacity.

Another interesting research area for VR technologies in health care are body swapping or assessments and treatments of eating disorders and obesities, anxiety disorders, stress related disorders, or pain management [16]. With regard to eating disorders and obesity, the objective of VR tools is to analyze effects by modifying the enduring memory of the body [42-44]. VR is assumed to integrate and extend existing treatments for eating and weight disorders (EWDs). The idea refers to alter the experience of the body (embodiment) in real time, e.g. in order to reduce food craving [45, 46]. Recent research suggests that the human brain and VR share the same basic mechanisms, i.e. the theoretical perspective of embodied simulations [47, 48]. According to neuroscientific research, the brain creates an embodied simulation of the body in the world to regulate and control the body in the world effectively. VR works in a similar way and tries to predict the sensory consequences of an individual's movements, providing its users the same scene they would see in the real world [49]. Here, like our brain, VR simulates a model of the body and the space around it and induces a high level of emotional engagement and sense of presence that can improve the chances of behaving different $[16,48,49]$.

Overall, many of these studies focus on opportunities for health outcomes or on the determination of the efficacy of VR treatments, therapies, and interventions. Here, mostly positive clinical effects are reported. However, limited attention is given to the technology and its characteristics itself, to underlying theories of its effectiveness, or to the interplay between both [14]. Moreover, current literature does not include adequate theoretical concepts and frameworks in order to achieve an improvement of desirable clinical outcomes. Consequently, we seek to review the literature and give an overview of current VR related research with regards to underlying theories as well as opportunities.

\section{Methods}

We conducted a literature review $[50,51]$ on the role of digital technologies, concerning VR related technologies such as VR, AR, and holography, in healthcare research and practice. For our methodical foundation, we deployed a scoping review as this type of review ensures a wide perspective on the research topic and provides a systematic orientation without lacking detailed insights of the included literature [52]. In order to reach this relatively broad scope but still ensure an adequate quality of our findings, we included studies published in international journals. We integrated comprehensive databases of both IS and healthcare/medical research to ensure examining the maximum number of relevant articles.

\subsection{Data Sources and Search Strategy}

Due to the novelty of Silva et al.'s article of 2018 [17] and their differentiation of VR, AR, MR, and MeR, we refer in our search spectrum to the distinction of VR and AR technologies as well as holography considering the fact that hitherto VR related technologies were mostly merged through VR and AR [53] or holographic technology [17] and the relative novel use and development of these technologies in health care. To find relevant articles for our objective, we searched the Pubmed, Web of Science, Livivo, CINAHL and IEEE databases. We used medical terms (medic* OR clinic* OR therap* OR surg* OR health) to combine them with technological terms (virtual* OR vr OR augment* OR ?dimens* OR hologr*) in a title search. We then added a keyword search with the terms (construct OR theor*) to focus our search on our research questions. As depicted above, we used the short form for virtual reality (VR), but left out the short form for augmented reality (AR) as it has too many different meanings in the medical discipline (e.g. allergic reaction or assisted respiration). We included English journal articles from all medical fields according to the purpose of our scoping review. There were no limits for the year of 
publication due to our extensive objective. Articles category were examined regarding their reference to theoretical foundations of the use and development of VR related technologies in medicine and health. The relevant content of the included articles were analyzed through full-text reading. Each step was conducted independently and afterwards matched to come to a joint result.

\subsection{Descriptive Results}

We identified 457 articles through our search. After removing duplicates, we screened title and abstract of 289 articles for their relevance. Following our initial research questions, we examined the relevance of these articles with regard to their theoretical foundations or implications. We excluded articles merely mentioning specific theories, but not discussing them explicitly in any part of the article. Furthermore, VR related technologies as defined above had to be an essential part in the study and had to take part in a direct clinical application or intervention. Therefore, 22 articles were included in assessment of full-text reading. Two of these articles were excluded due to eligibility. Finally, 20 articles were included in the theoretical meta-analysis. Figure 1 summarizes the process of literature search and our results [54].

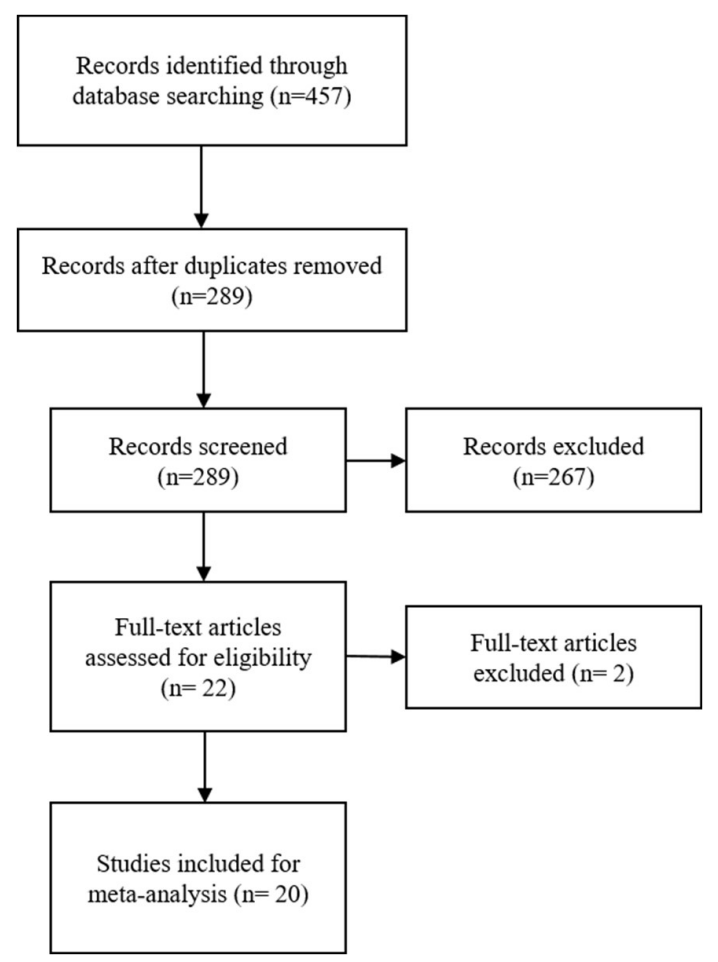

Figure 1. Process of study selection

\section{Findings}

In general, theoretical foundations are necessary to structure and accumulate scientific findings. Hence, a broader context can be created and new research can be informed [55]. In our findings, theoretical implications were mostly related to either IS-specific constructs or psychological theories. The ambiguity of theoretical foundation expresses itself in the manner of one-sided theoretical frames and a lack of synergistic approaches.

\subsection{Digital Technology in Health Care}

We considered three main developments of digital technology within our review: Virtual reality (VR), augmented reality (AR) and holographic technologies. In comparison, our findings suggest a thematic concentration on virtual and augmented reality in the medical field. No article concerning holographic interventions were found. In contrast, 17 articles concentrated on virtual reality, 1 on augmented reality and 2 mentioned both, virtual and augmented reality. From this unequal distribution of results, we deduce that a bigger part of medical research and practice concerns itself with current and future possible applications assisted by virtual reality and augmented reality technologies. Included reviews underline this hypothesis trough their thematic emphasis [14, 56, 57].

\subsubsection{Theoretical Foundation of VR related Technology in Health Care}

As VR and AR technologies have several practical capabilities, our findings show an ambiguous theoretical foundation in IS and healthcare research. Here, theoretical foundations are mainly used or discussed to explain the effectiveness of a VR-based therapeutic intervention. Therefore, most articles combine theoretical constructs of IS research with mid-range theories from psychology. Table 1 illustrates and summarizes all included articles of our search results. As depicted in Table 1, included articles are mainly focused on the empirical evaluation of VR-based interventions. Thereby, these articles refer to theoretical concepts and frameworks describing the mechanisms of clinical effectiveness. On the contrast, relatively few are concerned with the relationship between technological characteristics and participants' perception of the virtual worlds, i.e. technological effectiveness, or a combined perspective reflecting the relationship between technological characteristics and clinical effectiveness. Furthermore, relatively few articles focused on the conceptual or theoretical development with regards to our research objective. 
Table 1. Study design and theoretical perspective of included articles

\begin{tabular}{|c|c|c|c|}
\hline & \multicolumn{2}{|c|}{ Study Design } \\
\hline & & Empirical & Conceptual \\
\hline \multirow{3}{*}{$\begin{array}{l}\text { Theoretical } \\
\text { Perspective }\end{array}$} & $\begin{array}{l}\text { Clinical } \\
\text { Effectiveness }\end{array}$ & $\begin{array}{l}{[58],[59],[60],[61],[62],[14],[63],} \\
{[64],[65]}\end{array}$ & {$[66],[56]$} \\
\hline & $\begin{array}{l}\text { Technological } \\
\text { Effectiveness }\end{array}$ & [67], [68], [69] & [70], [71] \\
\hline & Combined & {$[57],[49]$} & [72], [73] \\
\hline
\end{tabular}

From our findings, there are mainly two key aspects concerning the theoretical foundation of VR related technologies in health care. Most of the included articles address psychological phenomena or analyze learning mechanisms. Considering how many medical fields are involved in health care and how many clinical studies are published each year, surprisingly few seem to relate their studies to basic theoretical assumptions. Findings concerning learning mechanisms refer to Skill Retention Theory [60], Cognitive Load Theory $[61,62]$ or combined different learning theories [59] while mentioning a broader theoretical basis. Skill Retention Theory basically allows to describe the process of learning procedures (in this case surgical skills). The study of Siu et al. [60] might lead to the assumption that VR related technologies are effective to learn new surgical skills and enable surgeons to refresh their own practical knowledge. A possible explanation for the improved effectiveness of learning based on the characteristics of VR related technologies is not mentioned and therefore a theoretical conclusion is missing. In a similar way, Cognitive Load Theory proposes that the success of learning is dependent on the amount of cognitive stress a person is exposed to while learning new skills. Interestingly, [62] and [61] come to different results: it appears to be unclear, if and how a VR simulation actually enhances or lowers the cognitive load of a person in comparison to a realworld medical procedure. Both studies do not make any conclusions for the theoretical understanding of the VR assisted process of learning. [59] used several theories of learning in combination (Situated Learning Theory, Experiential Learning Theory and Transformative Learning Theory) to build a framework for AR assisted education in health care explaining the effectiveness of AR assisted education with a positive effect induced by an extension of the real world. They conclude that AR is effective because of its ability to create a controllable learning environment and therefore help to increase a person's learning activity. Although [59] present a firm process of creating their theoretical framework, the theoretical implications concerning AR technologies are basically deduced from hypothetical characteristics of a specific AR system.

Aside from theories explaining processes of learning, [63] describe their theoretical fundament for VR-based exposure therapy by means of Emotional Processing Theory. This psychological theory hypothesizes that human fear or anxiety is manifested through mental structures. According to Emotional Processing Theory, an in vivo exposure might overstrain the resources of a patient, while avoidance of fear triggering situations does not change manifested fear structures. To overcome this problem, VR-based exposure offers a way to confront the patient enabling a higher level of situational control. [63] link immersion and presence to the characteristics of VR systems to legitimate their approach. Without explicitly mentioning these characteristics, [64] perform a comparable approach. Aside from the treatment of phobias or anxiety, [72] discussed the use of VR or AR based technology to assist personal change. Although [72] explain a detailed theoretical derivation of personal change with Perceptual Control Theory (this theory states that human behavior is the product of environmental adaptation) and SelfAffirmation Theory (this theory states that human behavior is a result of an intention to preserve selfintegrity), they find no approach to combine it with characteristics of VR or AR technologies. Similar, most of the included articles do not explicitly merge theoretical assumptions from a technological and psychological perspective. Furthermore, most of the included studies referring to psychological phenomena (clinical effectiveness perspective) legitimate or explain the effectiveness of VR related technologies for therapeutic use either from their empirical findings or from only hypothetical characteristics of those technologies.

It appears, only few studies explicitly combine theoretical assumptions from both the IS and psychological field. Often, VR related technologies are associated with basic cognitive functions that are involved in psychological therapies: exposition, 
distraction, motivation or engagement [56]. These functions are then associated with established constructs used in IS research, such as immersion or presence. In general, presence seems to be the most frequently used construct to merge psychological theories with characteristics of VR related technologies (see Table. 1). Thereby [70]'s conclusion of the reviewed literature is still accurate: research aims at the concept of presence, not at the cause of the effectiveness. Furthermore, several different definitions for presence exist, making it difficult for researchers to compare their findings [70]. Most of the articles including presence in their theoretical discussion include the construct of immersion as well. Although a distinction between these two terms are already extensively made in the literature (e.g., [74]), consistent definitions cannot be found within the included articles. As one possible distinction, [74] relate immersion to technological characteristics establishing an artificial environment. The underlying scale to evaluate the level of immersion is thereby linked to the similarity of the real world. Presence on the other hand describes a subjective, sensual perception of being in a remote environment [74]. As can be seen from the definitions of [74], one of these constructs might be desirable in a specific clinical setting, while the other might be not: e.g., in the study of [63], the authors conclude that presence might be helpful for participants to engage in an artificial situation where they are faced with triggers of their anxiety, while a high amount of immersion might lead to the perception of an actual real situation, resulting in a loss of control and decompensation of the patient. Therefore, to define adopted constructs is an important issue while contextualizing theoretical elements of two disciplines. [57].

Only two of our listed studies directly address this issue and manage to present a detailed synthesis of theoretical assumptions. [57] state that it is reasonable that VR related technologies have an actual effect on the therapeutic process, but "[d]espite these results, there is little theoretical and empirical understanding of how technologies actually support the users" (p.124). Therefore, [57] analyze the literature on the effectiveness of VR related technologies and conclude detailed characteristics from empirical studies. They manage to differentiate these characteristics into effects of VR related technologies on the client, the therapist and on the relationship of client and therapist. [57] then assign these characteristics into an existent model of the psychological therapy process. Even more detailed, [49] develop a model of embodied simulation to explain the effectiveness of VR on behavioral therapy. The occasion to develop a new model results from their analysis of the literature: "[a]n open issue not directly addressed by most of these articles is why VR is an effective clinical tool" (p.88). Therefore, the authors compare the construct of presence to basic brain functions. They state that "the more the VR model is similar to the brain model, the more the individual feels present in the VR world" (p.89). [49] then combine their definition of presence with Allocentric Lock Theory and argue that VR related technologies are effective to change the perception and behavior of patients because of their similarity to body-own simulations and, therefore, are able to change the self-perception of patients.

Overall, our findings suggest that the greater part of theoretical assumptions about the effectiveness of VR related technologies in health care actually results from experimental findings. Only few studies elaborate a theoretical model to base their interventions and interpretations on.

\section{Discussion and Implications}

In health care, VR related technologies are able to modify the relationship of patient and therapist as well as the process of therapy itself. From a theoretical perspective, it is important to understand why VR related technologies are effective. Therefore, already established theoretical elements from IS research, e.g. the construct or concept of presence, have to be merged with theories of clinical interventions. To reach this goal, such theoretical elements have to be put into context to establish valid models [75]. As clinical or psychological models of effectiveness already exist (e.g. the Allocentric Lock Theory or Skill Retention Theory), it appears to be reasonable to adopt important characteristics of technologies into these models by relating core constructs (like presence or immersion) to them. This way, constructs that are well established in IS research have to be linked to basic, central theoretical models, constructs or heuristics of clinical effectiveness to merge the gap between technological characteristics and the reason for their clinical effectiveness. In order to ensure comparability, core constructs thereby should be defined thoroughly.

From our findings, we conclude that only a small number of studies already made this effort to merge theoretical assumptions of two different disciplines into a composed model. Primarily, included studies either (i) took a theoretical framework for technological characteristics and measured an empirical effect of a specific technology without informing their framework with theoretical assumptions of clinical effectiveness (e.g., [69]) or (ii) explored the theoretically legitimated clinical effectiveness of a specific technology in comparison 
to a standard therapy without informing their framework with theoretical elements of technology characteristics (e.g., [60]). Both, (i) and (ii) bear the risk of modelling an intervention not the best way possible, especially from the perspective of a patient or client. Additionally, to overcome the central gap in research to explain why a specific VR-based intervention might be effective, theories or their development are needed. This holds especially for clinical disciplines aside psychology (e.g., surgery), where theoretical foundations appear to be in an earlier stage compared to VR related psychological interventions.

Concerning already existent approaches, VR related technologies are capable of extending the real world through creation of artificial situations, but simultaneously are capable to constrain it. That enables therapists to create controllable situations, which might lead to an advantage in specific therapeutic settings. E.g., VR related technologies seem to be a chance for psychological therapy where an in vivo exposure is not indicated, but patients successfully managed to cope within imaginative therapeutic sessions. Therefore, presence seems to be an important construct that affect therapeutic outcomes. It reflects mechanisms of directing attention or distraction, which are fundamental for VR [71]. But as [70] mentioned, the fundaments of its effectiveness remain still unclear. Moreover, theoretical foundations of VR related technologies are often empirically derived from or applied to psychological low and midrange theories. They often adapt the nature of those psychological theories in terms of mere descriptions or focus on the interaction of related constructs. There is still a lack of wide-range theories, derived from grand social or psychological theories, and a lack of synthesized, empirically explored and tested theories. Furthermore, a more detailed description and derivation of such theoretical approaches are helpful to understand the underlying assumptions of a hypothetical effectiveness of VR-based technologies.

Nevertheless, our findings are subject to some limitations. Due to our broad search strategy and our chosen type of review, we did not assess the methodological and overall quality of included articles resulting in a less differentiated synthesis of our findings. In addition, this study primarily aims at the completeness of searching, rather than an in-depth analysis of the subject. For future research, an in-depth analysis of specific theoretical constructs and their relatedness to clinical outcomes might provide comprehensive frameworks to further guide clinical interventions involving VR related technologies. In this regard, our results encourage the field of IS to increase their participation in the theme of VR related technologies in health care. To overcome mere descriptions of technological characteristics serving as theoretical legitimation, more complex and detailed approaches combining constructs such as presence or immersion with primary and secondary patient-related outcomes are needed. Lastly, our review was conducted in the field of health care, resulting in limited generalizability. Future research might broaden the spectrum of disciplines to explore contextual and general findings concerning the theoretical foundations of VR related technologies and their effectiveness.

\section{Conclusion}

Our results provide an overview of existing theoretical foundations of use and development for VR related technology in health care. We explored what kind of theoretical combinations or synergetic approaches already exist in the field and what kind of approaches are needed to expand our knowledge about the effectiveness of VR related technologies in health care. This appears highly relevant as the development of clinical VR applications based only on heuristics or previous non-theoretical research might lead to a focus on the effect of clinical VR use itself (outcome-based research), whereas theories of effectiveness are able to guide and filter ideas of research and phenomena. The latter seems to be crucial to understand undergoing mechanisms of VR-based therapeutic processes. In our review, we primarily discussed theoretical aspects of effectiveness. To widen the scope of understanding, practical contexts of VR related technologies could be brought into focus, e.g. by analyzing, in what kind of clinical disciplines those technologies are used in general and what outcomes are targeted.

From all theoretical foundations that were discussed in this paper, only a few are able to provide a basis to build on future research and to explain technology mediated effectiveness of clinical interventions. Considering possible fields of application in health care (education, psychology, surgery, rehabilitation, etc.) and empirical indications of effectiveness, the high potential of VR related technologies benefits from theoretical foundations to structure ongoing research and hold explanations for underlying relations. Therefore, further studies may provide more complex theoretical models to enrich insights on the role of VR related technologies for health care. 


\section{References}

[1] Tang R, Yang X-D, Bateman S, Jorge J, Tang A Physio@Home: Exploring Visual Guidance and Feedback Techniques for Physiotherapy Exercises. In: Begole B, Kim J, Inkpen $\mathrm{K}$, Woo $\mathrm{W}$, editors. Physio@Home: Exploring Visual Guidance and Feedback Techniques for Physiotherapy Exercises; 2015. New York, New York, USA: ACM Press; 412332.

[2] Wilson N, Couper I, Vries E, Reid S, Fish T, Marais B. A critical review of Interventions to redress the inequitable distribution of healthcare professionals to rural and remote areas. Rural and remote health 2009; 9.

[3] Martínez A, Villarroel V, Seoane J, Pozo FD. Rural telemedicine for primary healthcare in developing countries. IEEE Technology and Society Magazine 2004; (23): 13-22.

[4] Mutter D, Bouras G, Marescaux J. Digital technologies and quality improvement in cancer surgery. Eur J Surg Oncol 2005; 31(6): 689-94

[5] Hollis C, Morriss R, Martin J, et al. Technological innovations in mental healthcare: harnessing the digital revolution. Br J Psychiatry 2015; 206(4): 263-5

[6] Kvedar J, Coye MJ, Everett W. Connected health: a review of technologies and strategies to improve patient care with telemedicine and telehealth. Health Aff (Millwood) 2014; 33(2): 194-9

[7] Pantelopoulos A, Bourbakis NG. A Survey on Wearable Sensor-Based Systems for Health Monitoring and Prognosis. Systems, Man, and Cybernetics, Part C: Applications and Reviews, IEEE Transactions on 2010; 40

[8] Reger GM, Holloway KM, Candy C, et al. Effectiveness of virtual reality exposure therapy for active duty soldiers in a military mental health clinic. Journal of traumatic stress 2011; 24(1): 93-6

[9] Chaudhry B, Wang J, Wu S, et al. Systematic review: impact of health information technology on quality, efficiency, and costs of medical care. United States; 2006.

[10] Chan S, Conti F, Salisbury K, Blevins NH. Virtual reality simulation in neurosurgery: technologies and evolution. Neurosurgery 2013; 72 Suppl 1: 154-64

[11] Davis A, Murphy J, Owens D, Khazanchi D, Zigurs I. Avatars, People, and Virtual Worlds: Foundations for Research in Metaverses. Journal of the Association for Information Systems 2009; (10 (2)).

[12] Hwang W-Y, Hu S-S. Analysis of peer learning behaviors using multiple representations in virtual reality and their impacts on geometry problem solving. Computers \& Education 2013; 62: 308-19

[13] Slater M, Sanchez-Vives MV. Enhancing Our Lives with Immersive Virtual Reality. Front. Robot. AI 2016; 3: 751

[14] Garrett B, Taverner T, Gromala D, Tao G, Cordingley E, Sun C. Virtual Reality Clinical Research: Promises and Challenges. JMIR serious games 2018; 6(4)

[15] Guyot J-P, Perez Fornos A, Guinand N, van de Berg R, Stokroos R, Kingma H. Vestibular assistance systems: promises and challenges. J Neurol 2016; 263(Suppl 1): S30-5

[16] Riva G, Wiederhold BK, Gaggioli A. Being Different: The Transformative Potential of Virtual Reality. Annual Review of Cyber Therapy and Telemedicine 2016; (14): 26-9.

[17] Silva JNA, Southworth M, Raptis C, Silva J. Emerging Applications of Virtual Reality in Cardiovascular Medicine. JACC Basic Transl Sci 2018; 3(3): 420-30

[18] Cipresso P, Giglioli IAC, Raya MA, Riva G. The Past, Present, and Future of Virtual and Augmented Reality Research: A Network and Cluster Analysis of the Literature. Frontiers in psychology 2018; 9: 2086

[19] Nitschke P, Bork U, Plodeck V, et al. Importance of preoperative and intraoperative imaging for operative strategies. Chirurg 2016; 87(3): 179-88

[20] Botella C, Fernandez-Alvarez J, Guillen V, GarciaPalacios A, Banos R. Recent Progress in Virtual Reality Exposure Therapy for Phobias: A Systematic Review. Current Psychatrie Reports 2017; 19(7)

[21] Crosbie JH, Lennon S, Basford JR, McDonough SM. Virtual reality in stroke rehabilitation: still more virtual than real. Disabil Rehabil 2007; 29(14): 1139-46; discussion 1147-52

[22] Mills S, Noyes J. Virtual reality: an overview of Userrelated Design Issues. Interacting with Computers 1999; 11(4): 375-86

[23] Khalifa M, Shen N. System Design Effects on Social Presence and Telepresence in Virtual Communities 2004.

[24] Martín-Gutiérrez J. Virtual Technologies Trends in Education. Eurasia J. Math. Sci. Tech. Ed 2017; 13(1)

[25] Seibert J, Shafer DM. Control mapping in virtual reality: effects on spatial presence and controller naturalness. Virtual Reality 2018; 22(1): 79-88

[26] Wexelblat A, editor. Virtual reality: Applications and explorations. 2. [Dr.] Boston: Academic Press Professional; 1993.

[27] Morris LD, Louw QA, Crous LC. Feasibility and potential effect of a low-cost virtual reality system on reducing pain and anxiety in adult burn injury patients during physiotherapy in a developing country. Burns 2010; 36(5): 659-64

[28] Nilsson A, Axelsson A-S, Heldal I, Schroeder R. The Long-term Uses of Shared Virtual Environments: An Exploratory Study. In: Diaper D, Sanger C, Schroeder $\mathrm{R}$, editors. The Social Life of Avatars. London: Springer London 2002; 112-26.

[29] Schultze U. Embodiment and Presence in Virtual Worlds: A Review. Journal of Information Technology 2010; 25(4): 434-49

[30] Müns A, Meixensberger J, Lindner D. Evaluation of a novel phantom-based neurosurgical training system. Surg Neurol Int 2014; 5: 173

[31] Chen M, Lin H. Virtual geographic environments (VGEs): originating from or beyond virtual reality (VR)? International Journal of Digital Earth 2018; 11(4): 329-33

[32] Lin H, Chen M, Lu G, et al. Virtual Geographic Environments (VGEs): A New Generation of 
Geographic Analysis Tool. Earth-Science Reviews 2013; 126: 74-84

[33] Lartigue J, Scoville T, Pham M. Promoting K-8 Learning using Oculus Rift: Employing Virtual Reality to Increase Learning Outcomes in Elementary Biology. In: Bastiaens T, editor. E-Learn: World Conference on E-Learning in Corporate, Government, Healthcare, and Higher Education 2014. New Orleans, LA, USA: Association for the Advancement of Computing in Education (AACE) 2014; 1100-5.

[34] Tang S-L, Kwoh C-K, Teo M-Y, Sing NW, Ling K-V. Augmented reality systems for medical applications. IEEE Engineering in Medicine and Biology Magazine 1998; 17(3): 49-58

[35] Lucile Packard Children's Hospital Stanford pioneers use of VR for patient care, education and experience; 2017 [cited 2019 April 26]

[36] Lubinger B, Guhl Hammer J. Case Western Reserve, Cleveland Clinic Collaborate with Microsoft on 'EarthShattering' Mixed-Reality Technology for Education; 2015. Available from: URL: https://case.edu/hololens/.

[37] Sundeep M. Hologram the future of medicine - From Star Wars to clinical imaging. Indian Heart J 2017; 69(4): 566-7

[38] Chan FP, Aguirre S, Bauser-Heaton H, Hanley F, Perry SB. Head Tracked Stereoscopic Pre-surgical Evaluation of Major Aortopulmonary Collateral Arteries in the Newborns. Chicago IL. 2013 Dec 1.

[39] Bruckheimer E, Rotschild C, Dagan T, et al. Computergenerated real-time digital holography: first time use in clinical medical imaging. Eur Heart $\mathrm{J}$ Cardiovasc Imaging 2016; 17(8): 845-9

[40] Silva JN, Southworth MK, Dalal A, van Hare GF, Silva JR. Abstract 15358: Improving Visualization and Interaction During Transcatheter Ablation Using an Augmented Reality System: First-in-Human Experience. Circulation 2017; 136(suppl_1): A15358A15358

[41] Kress B, Shin M. Diffractive and holographic optics as optical combiners in head mounted displays. In: Mattern F, Santini S, Canny JF, Langheinrich M, Rekimoto J, editors. Diffractive and holographic optics as optical combiners in head mounted displays; 2013. New York, New York, USA: ACM Press; 1479-82.

[42] Corno G, Serino S, Cipresso P, Baños RM, Riva G. Assessing the Relationship Between Attitudinal and Perceptual Component of Body Image Disturbance Using Virtual Reality. Cyberpsychology, behavior and social networking 2018; 21(11): 679-86

[43] Serino S, Pedroli E, Keizer A, et al. Virtual Reality Body Swapping: A Tool for Modifying the Allocentric Memory of the Body. Cyberpsychology, behavior and social networking 2016; 19(2): 127-33

[44] Serino S, Polli N, Riva G. From avatars to body swapping: The use of virtual reality for assessing and treating body-size distortion in individuals with anorexia. J Clin Psychol 2019; 75(2): 313-22

[45] Gutiérrez-Maldonado J, Wiederhold BK, Riva G Future Directions: How Virtual Reality Can Further Improve the Assessment and Treatment of Eating
Disorders and Obesity. Cyberpsychology, behavior and social networking 2016; 19(2): 148-53

[46] Wiederhold BK, Riva G, Gutiérrez-Maldonado J. Virtual Reality in the Assessment and Treatment of Weight-Related Disorders. Cyberpsychology, behavior and social networking 2016; 19(2): 67-73

[47] Riva G. The neuroscience of body memory: From the self through the space to the others. Cortex 2018; 104: 241-60

[48] Riva G, Dakanalis A. Altered Processing and Integration of Multisensory Bodily Representations and Signals in Eating Disorders: A Possible Path Toward the Understanding of Their Underlying Causes. Front Hum Neurosci 2018; 12: 49

[49] Riva G, Wiederhold BK, Mantovani F. Neuroscience of Virtual Reality: From Virtual Exposure to Embodied Medicine. Cyberpsychology, behavior and social networking 2019; 22(1): 82-96

[50] Boell SK, Cecez-Kecmanovic D. On being 'Systematic' in Literature Reviews in IS: Debates and Perspectives. Journal of Information Technology 2015; 30(2): 161-73

[51] Vom Brocke J, Simons A, Riemer K, Niehaves B, Plattfaut R, Cleven A. Standing on the Shoulders of Giants: Challenges and Recommendations of Literature Search in Information Systems Research. CAIS 2015; 37

[52] Grant MJ, Booth A. A typology of reviews: an analysis of 14 review types and associated methodologies. Health Info Libr J 2009; 26(2): 91-108

[53] Milgram P, Kishino F. A Taxonomy of Mixed Reality Visual Displays. IEICE Transactions on Informations Systems 1994; E77-D(12): 1321-9.

[54] Moher D, Liberati A, Tetzlaff J, Altman DG, The PRISMA Group. Preferred Reporting Items for Systematic Reviews and Meta-Analyses: The PRISMA Statement. PLOS Medicine 2009; 6(7): 1-6

[55] Gregor. The Nature of Theory in Information Systems. MIS Quarterly 2006; 30(3): 611

[56] Rizzo AS, Koenig ST. Is clinical virtual reality ready for primetime?; 2017. Available from: URL: https://www.researchgate.net/publication/319165844_ Is_Clinical_Virtual_Reality_Ready_for_Primetime.

[57] Wrzesien $\bar{M}$, Burkhardt J-M, Botella $\bar{C}$, Alcaniz M. Towards a Virtual Reality- and Augmented RealityMediated Therapeutic Process model: a theoretical revision of clinical issues and HCI issues. Theoretical Issues in Ergonomics Science 2015; 16(2): 124-53

[58] Johnson S, Price M, Mehta N, Anderson PL. Stereotype confirmation concerns predict dropout from cognitive behavioral therapy for social anxiety disorder. BMC Psychiatry 2014; 14: 233

[59] Zhu E, Lilienthal A, Shluzas LA, Masiello I, Zary N. Design of Mobile Augmented Reality in Health Care Education: A Theory-Driven Framework. JMIR medical education 2015; 1(2)

[60] Siu K-C, Best BJ, Kim JW, Oleynikov D, Ritter FE. Adaptive Virtual Reality Training to Optimize Military Medical Skills Acquisition and Retention. Military medicine 2016; 181 $(5, \mathrm{~S}): 214-20$ 
[61] Andersen SAW, Mikkelsen PT, Konge L, CayeThomasen P, Sorensen MS. Cognitive load in distributed and massed practice in virtual reality mastoidectomy simulation. Laryngoscope 2016; 126(2): E74-9

[62] Rasmussen SR, Konge L, Mikkelsen PT, Sorensen MS, Andersen SAW. Notes From the Field: Secondary Task Precision for Cognitive Load Estimation During Virtual Reality Surgical Simulation Training. Eval Health Prof 2016; 39(1): 114-20

[63] Wilhelm FH, Pfaltz MC, Gross JJ, Mauss IB, Kim SI, Wiederhold BK. Mechanisms of virtual reality exposure therapy: the role of the behavioral activation and behavioral inhibition systems. Appl Psychophysiol Biofeedback 2005; 30(3): 271-84

[64] Rizzo A, Parsons TD, Lange B, et al. Virtual Reality Goes to War: A Brief Review of the Future of Military Behavioral Healthcare. Journal of Clinical Psychology in Medical Setings 2011; 18(2): 176-87

[65] Gorrindo T, Groves JE. The psychodynamics of transference--a virtual reality model. Am J Psychother 2012; 66(2): 151-63

[66] Riva G. The key to unlocking the virtual body: virtual reality in the treatment of obesity and eating disorders. J Diabetes Sci Technol 2011; 5(2): 283-92

[67] Viciana-Abad R, Reyes-Lecuona A, García-Berdones C, Díaz-Estrella A. A preliminary study of presence in virtual reality training simulation for medical emergencies. Studies in health technology and informatics 2004; 98: 394-6.

[68] Price M, Anderson P. The role of presence in virtual reality exposure therapy. Journal of Anxiety Disorders 2007; 21(5): 742-51
[69] Londero A, Viaud-Delmon I, Baskind A, et al. Auditory and visual 3D virtual reality therapy for chronic subjective tinnitus: theoretical framework. Virtual Reality 2010; 14(2): 143-51

[70] Schuemie MJ, van der Straaten P, Krijn M, van der Mast, C A. Research on presence in virtual reality: a survey. Cyberpsychology \& behavior: the impact of the Internet, multimedia and virtual reality on behavior and society $2001 ; 4(2)$ : 183-201

[71] Riva G, Molinari E, Vincelli F. Interaction and presence in the clinical relationship: Virtual reality (VR) as communicative medium between patient and therapist. IEEE Transaction on Information Technology in Biomedicine 2002; 6(3): 198-205

[72] Riva G, Baños RM, Botella C, Mantovani F, Gaggioli A. Transforming Experience: The Potential of Augmented Reality and Virtual Reality for Enhancing Personal and Clinical Change. Frontiers in Psychiatry 2016; 7

[73] Mohr DC, Burns MN, Schueller SM, Clarke G, Klinkman M. Behavioral intervention technologies: evidence review and recommendations for future research in mental health. Gen Hosp Psychiatry 2013; 35(4): 332-8

[74] Slater M, Wilbur S. A Framework for Immersive Virtual Environments (FIVE): Speculations on the Role of Presence in Virtual Environments. Presence 1997; 6(6): 603-16

[75] Hong W, Chan FKY, Thong JYL, Chasalow LC, Dhillon G. A Framework and Guidelines for ContextSpecific Theorizing in Information Systems Research. Information Systems Research 2014; 25(1): 111-36 